\title{
Recent Advances in Biomarkers and Potential Targeted Therapies in Head and Neck Squamous Cell Carcinoma
}

\author{
Eric J. Yavrouian and Uttam K. Sinha \\ Department of Otolaryngology, Keck School of Medicine of USC, University of Southern California, Los Angeles, CA 90089, USA \\ Correspondence should be addressed to Uttam K. Sinha, sinha@usc.edu
}

Received 22 August 2011; Accepted 26 September 2011

Academic Editor: D. E. Ziogas

Copyright (C 2012 E. J. Yavrouian and U. K. Sinha. This is an open access article distributed under the Creative Commons Attribution License, which permits unrestricted use, distribution, and reproduction in any medium, provided the original work is properly cited.

\begin{abstract}
Head and neck squamous cell carcinoma (HNSCC) is a devastating tumor of the upper aerodigestive tract with no significant change in treatment modality or improvement in survival over the last several decades. Biomarkers are important biological molecules that can be utilized in tumor detection, prognosis, and as targeted therapies. There are several important biomarkers and potential targets in the forefront, including biomarkers of tumorigenesis, signal transduction molecules, proteins involved in angiogenesis, and oncogenic viruses. The clinical applications of these biomarkers are in various states from in vitro and in vivo models, phase II and III clinical trials, to accepted modes of treatment in patients with HNSCC. Given the potential improvement in prognosis that biomarkers and their targeted therapies may have on the treatment of HNSCC, their investigation is both important and essential.
\end{abstract}

\section{Introduction}

Squamous cell carcinoma is the most common cancer arising in the head and neck with devastating effects on communication, swallowing, and, most importantly, survival. Head and neck squamous cell carcinoma (HNSCC) is an epithelial cancer arising in the upper aerodigestive tract for which new biomarkers and targeted therapies are needed for rapid diagnosis and treatment. It is a tumor occurring most commonly in the oral cavity, oropharynx, hypopharynx, and larynx [1]. In 2000, there were 480,000 new cases of HNSCC each year worldwide and HNSCC compromised approximately $4 \%$ of malignant neoplasms $[2,3]$. In the United States, approximately 36,000 cases are expected to occur in 2010 with an estimated 8,000 deaths [4]. Men are at greater risk than women with the two greatest risk factors consistently being tobacco and alcohol use. Traditionally, the major treatment for HNSCC has been surgical resection with postoperative chemoradiation. This treatment modality has not significantly changed over the past 30 years with only minimal improvement in survival. Overall survival ranges from 70 to $85 \%$ for patients presenting with early-stage disease (stage I and II) to $30-40 \%$ for advanced-stage disease (stages III and
IV) [5]. Thus, new biomarkers for earlier detection as well as targeted therapies are essential.

Tumor biomarkers are a novel avenue with which one can improve early detection of tumor, improve monitoring and treatment, and ultimately increase disease survival. Biomarkers are biological molecules that when measured can correlate with the presence or absence of primary disease and metastasis, predict disease prognosis, or offer a potential target for specified therapies. A diverse range of biomarker types exist and can be measured including changes in the host genome, differential expression of proteins involved in processes such as angiogenesis, and the presence of viral infections. In recent years, several new biomarkers have been identified and are currently being studied for their effectiveness in HNSCC detection, prognosis, and treatment.

\section{Tumor Pathogenesis}

Neoplasia in the head and neck is a multistep process with sequential mutations in genes responsible for tumor surveillance. A microsatellite analysis of allelic alterations demonstrated that with the accumulation of genetic mutations, one 
can follow the transformation from simple squamous hyperplasia to severe dysplasia, and, ultimately, invasive squamous cell carcinoma [6]. p53, a tumor suppressor gene, has been implicated in the early pathogenesis of HNSCC, as it controls cell growth through regulation of the cell-cycle and apoptosis [7-9]. In a study analyzing HNSCC patients with a history of tobacco and alcohol use, Brennan et al. found a significantly higher proportion of patients with mutations of p53 and other distinct sites when compared to nonsmokers and nondrinkers [10]. p53 mutations have been found in up to $50 \%$ of HNSCC patients and have been shown to be associated with decreased survival [8].

In addition to $\mathrm{p} 53$, mutations in the retinoblastoma $(\mathrm{Rb})$ gene are involved in the pathogenesis of HNSCC. p16 ${ }^{\mathrm{INK} 4 \mathrm{~A}}$, a major target of the Rb pathway, is a tumor suppressor gene; its function is inhibited through a variety of pathways including loss of heterozygosity $(\mathrm{LOH})$ of chromosome 9p21 where it is located. $\mathrm{LOH}$ of $9 \mathrm{p} 21$ is seen in $30 \%$ of premalignant lesions and up to $80 \%$ of malignant lesions [11]. $\mathrm{LOH}$ in other locations, including $\mathrm{LOH}$ of chromosome $3 \mathrm{p}$, has also been associated with tumorigenesis. Lee et al. examined mutations in eight different HNSCC cell lines and found that three candidate oncogenes encoded on chromosome $3 \mathrm{p}$ (ALS2CL, EPHA3, and CMYA1) were mutated, implying that $\mathrm{LOH}$ of chromosome $3 \mathrm{p}$ is also associated with HNSCC [12]. An ongoing clinical trial of the tyrosine kinase inhibitor Erlotinib and oral cancer is assessing the clinical outcomes in patients with $\mathrm{LOH}$ of $9 \mathrm{p}$ and $3 \mathrm{p}$ compared to patients with normal DNA [13]. LOH of both $3 p$ and $9 p$ has also been shown to help differentiate dysplastic and hyperplastic lesions that are likely to progress to carcinoma $[11,14,15]$. Despite the fact that p53 and Rb mutations as well as $\mathrm{LOH}$ at $3 p$ and $9 p$ are clinically relevant biomarkers of HNSCC, they have yet to be applied in daily practice.

In addition to the p53 and $\mathrm{Rb}$ genes, sphingosine kinases (SphKs) have recently emerged as molecules of interest in HNSCC. By regulating levels of ceramide, sphingosine, and sphingosine-1-phosphate (S1P), SphK influences cells to enter proliferative states as opposed to apoptotic states [1619]. SphK1, the SphK isozyme most studied in neoplastic diseases, has been shown to be upregulated in HNSCC with overexpression in advanced stage and recurrent tumors. Use of small molecular inhibitors or siRNA's targeting SphK1 has been demonstrated to sensitize cells both in in vitro and in vivo studies to radiation $[20,21]$. As a cell cycle regulator overexpressed in HNSCC patients, SphK1 provides another prospective avenue in the treatment of HNSCC.

\section{Signal Transduction Biomarkers}

One of the most well-known biomarkers in HNSCC is the Epidermal Growth Factor Receptor (EGFR). EFGR is a receptor tyrosine kinase involved in multiple downstream signaling pathways influencing cell growth, angiogenesis, and invasion [22]. Downstream EGFR signaling activates the mitogen-activated protein kinase (MAPK) pathway as well as the phosphatidylinositol 3-kinase (PI3-K)/protein kinase B (Akt) pathway [23]. Activation of the MAPK pathway leads to increased expression of antiapoptotic proteins like $\mathrm{Bcl}-\mathrm{x}_{2}$ and inhibition of proapoptotic proteins like BAD [24]. Signaling through the PI3-K/Akt pathway ultimately leads to inhibition of the tumor suppressor gene p53 [25]. All of these result in a proliferative state and inhibition of tumor suppressor function.

Overexpression of EGFR in HNSCC has been associated with poorer overall survival and recurrence, and up to $90 \%$ of HNSCC patients express EGFR [22, 26-28]. With EGFR overexpression implicating a poor prognosis, it was one of the first biomarkers targeted as a potential therapy for HNSCC. Cetuximab, a monoclonal antibody directed against the extracellular receptor domain of EGFR, blocks ligand binding and subsequent downstream signaling, in addition to its role in the long-term downregulation of the receptor expression [29-33]. It has been the most successful targeted therapy applied in HNSCC to date. In a phase III clinical trial by Bonner et al., cetuximab in combination with radiotherapy provided an overall survival benefit of an additional 20 months compared to radiation alone [34, 35]. There have also been several clinical trials comparing chemotherapy alone or in combination with cetuximab. In the phase III trial, Erbitux in first-line treatment of recurrent or metastatic head and neck cancer (EXTREME), 442 patients with recurrent or metastatic HNSCC were randomized to receive either platinum/5-FU alone or cetuximab plus platinum/5FU. Results showed an increase in response rate from $20 \%$ in the chemotherapy group to $36 \%$ in the chemotherapy plus cetuximab group, with an overall survival increase from 7.4 months in the chemotherapy group to 10.1 months in the chemotherapy and cetuximab group [36]. Another phase III clinical trial from the Radiation Therapy Oncology Group (RTOG) comparing concurrent chemoradiation and Cisplatin versus concurrent chemoradiation with Cisplatin and cetuximab in patients with stage III and IV HNSCC is pending [13].

In addition to monoclonal antibodies targeting EGFR, small molecule tyrosine kinase inhibitors (TKIs) are also capable of inhibiting EGFR function. Phase I and II clinical trials in patients with recurrent or metastatic HNSCC have been conducted on the TKIs erlotinib and gefitinib. With erlotinib, these trials show an overall survival range of six to eight months and a response rate from $4 \%$ to $21 \%$. With gefitinib, phase I and II clinical trials have demonstrated an overall survival ranging from six to eight months, with a response rate of $1 \%$ to $15 \%$ [37-41]. While studies of erlotinib and gefitinib have demonstrated some response in HNSCC, results of phase III clinical trials on TKIs are still pending [13].

\section{Biomarkers of Angiogenesis}

In addition to their potential as useful biomarkers in HNSCC, markers of angiogenesis provide a therapeutic opportunity in HNSCC. Angiogenesis play an important role in tumor growth and progression [42]. Without new vessel growth, tumors are unlikely to grow beyond $3 \mathrm{~mm}$ [43-46]. There are three families of receptor tyrosine kinases involved in angiogenesis: the ephrins and the Eph receptors, the angiopoetin family, and the vascular endothelial growth factor 
(VEGF) [47]. Of these three receptor tyrosine kinase families, VEGF is the most extensively studied. VEGF has been shown to be overexpressed in tumor cells compared to normal cells; this overexpression is associated with a 1.88 -fold increased risk of death and is also associated with lymph node metastasis [48-50].

There are several antiangiogenic targets currently undergoing clinical trial. Tyrosine kinase inhibitors of the VEGF receptors halt their intracellular signaling. Several of these small molecule tyrosine kinase inhibitors, sunitinib, sorafenib, vandetanib, semaxanib, and foretinib, are undergoing phase II clinical trials [13]. Sunitinib was studied by Machiels et al. in a phase II clinical trial of 38 HNSCC patients in which it was given as a palliative treatment; they achieved a disease control rate of 50\%. However, due to several complications that occurred including bleeding, skin ulceration, and fistulas, they recommended further study of the drug to assess which patients would benefit [51]. Sorafenib's effect was studied in recurrent/metastatic HNSCC and nasopharyngeal carcinoma with a response rate of $3.7 \%$. Given that it is a multikinase inhibitor, its effect cannot be attributed only to its antiangiogenic activity [52]. Semaxanib was also studied in HNSCC as a single agent, but its use was discontinued due to several adverse affects and its difficulty with administration [53]. Bevacizumab, a monoclonal antibody against the VEGFA ligand, has been reviewed in phase I and II clinical trials with convincing evidence of antitumor activity in HNSCC patients when combined with erlotinib $[54,55]$. Vandetanib, a VEGF receptor inhibitor, is one of the drugs currently undergoing clinical trial. This drug is unique in that it acts as an inhibitor of the VEGF receptor, the EGFR receptor, and the rearranged during transfection (RET) tyrosine kinases. In vitro, it has an inhibitory effect on HNSCC cells; however, results of phase II clinical trials are currently under investigation $[13,56]$.

Another receptor tyrosine kinase of potential interest in HNSCC is the Eph receptor family and its ligands, the Ephrins. This group of proteins has an important role in many physiologic processes including cell aggregation and migration, angiogenesis, and vascular network development [57-59]. EphB4 and its sole ligand EphrinB2 are overexpressed in all primary and metastatic tumors, with EphB4 overexpression correlating with advanced stage disease and lymph node metastasis. In vivo, EphB4 has also been demonstrated to provide a survival advantage to tumor cells, and, its inhibition has been shown to decrease the survival of the HNSCC tumor cells. Furthermore, an analysis of HNSCC patients and EphB4/EphrinB2 expression demonstrated that overexpression of EphB4 and EphrinB2 was associated with a significantly worse overall survival [60-63]. Given that EphB4 and EphrinB2 are overexpressed in HNSCC and that this is associated with worse overall survival, EphB4 and EphrinB2 are potentially useful biomarkers and may provide another target for HNSCC treatment.

\section{Oncogenic Viruses}

In recent years, the human papilloma virus (HPV) and its link with HNSCC, particularly in oropharyngeal tumors, has been illustrated. Not only can HPV be used as a biomarker of prognostic significance, but also as a preventative target. Of the several types of HPV, type 16 is most commonly associated with HNSCC [64-66]. HPV is a double-stranded DNA virus that encodes several proteins, among which are three oncoproteins: E5, E6, and E7 [67]. The carcinogenic effect of HPV is mainly due to oncoproteins E6 and E7. HPV E6 expression ultimately leads to disruption in function of p53 and its antitumor protective effect [68]. Similarly, oncoprotein $\mathrm{E} 7$ inhibits the $\mathrm{Rb}$ protein and its tumor suppressor function [69].

Using either PCR or in situ hybridization assays, HPV status can be detected in head and neck tumors $[65,70]$. The RTOG 0129 clinical trial showed that $64 \%$ of oropharyngeal tumors were HPV-positive and that this subset of patients was younger, less likely to use tobacco, and had smaller tumors [71]. Thus, HPV-positive HNSCC patients have a significantly improved survival and treatment outcome independent of the mode of treatment [72-74]. In a prospective study evaluating HNSCC patients treated with induction chemotherapy and radiation, patients with HPV-positive tumors had a higher response after treatment, $84 \%$ in the HPV-positive group versus $57 \%$ in the HPV negative tumors. Two-year overall survival in patients with HPV-positive tumors was $95 \%$ compared to $62 \%$ in patients who were HPV negative [72].

HPV status can be used as a biomarker of improved prognosis; however, it also has a potential application in HNSCC management, both with preventative vaccination and as a targeted therapy. In 2006, the HPV vaccine, Gardasil was approved by the US Food and Drug Administration and recommended by the Center for Disease Control (CDC) in girls and young women to prevent cervical cancer. The vaccine targets HPV types 6, 11, 16, and 18. Since then, in 2009, the CDC has also recommended the vaccine in young men aged nine through 26 for the prevention of genital warts and HPV-associated cancers including HNSCC [75]. In an effort to utilize HPV status as a mode of treatment, Wu et al. have developed an HPV vaccine that enhances the T-cell immune response in mice with HPV-positive tumors [76]. This vaccine has potential as a therapeutic modality in patients with HPV-positive tumors to further improve survival.

\section{Biomarkers in Saliva}

With advances in the search for biomarkers in HNSCC, many researchers are interested in finding potential biomarkers in one of the most easily accessible tissues, patients' salivamainly through the study of differential gene expression, expression of proteins such as telomerase, and through mass spectrometry. In a study reviewing 82 candidate genes, Sethi et al. were able to demonstrate the differential expression of genes present in the saliva of HNSCC patients as compared to controls. The expression of the genes, PMAIP1 and PTPN1, correlated with HNSCC in 27 patients compared to 10 control patients [77]. Telomerase, a protein involved in emergence from cellular senescence, has an increased degree of activity in malignant cells. With PCR-based techniques using oral rinses from HNSCC patients, Califano et al. was able to 
show increased telomerase activity in the saliva of HNSCC patients compared to controls [78]. In recent years, with advances in the field of proteomics and mass spectrometry, one is able to simultaneously analyze multiple proteins found in oral rinses in an attempt to discover potential tumor biomarkers [79]. There is a significant interest in developing a study model with which HNSCC can be detected in saliva, however, one biomarker or method of detection has yet to be applied systemically outside of the laboratory setting.

\section{Discussion}

With further advances in our understanding of HNSCC and its pathogenesis, HNSCC is a seemingly heterogeneous group of tumors rather than a single type with one appropriate treatment. With the emergence of several new biomarkers, one can improve detection of tumor, obtain prognostic information and offer new treatments in head and neck squamous cell carcinoma. As we delineate which biomarkers are overexpressed in patients, we can offer individual patients with HNSCC-specific prognostic information, and tailor treatments to patients based on the molecular profile of their tumor.

To date, few of these biomarkers are applied clinically for prognostic information. Although in many studies the overexpression of these biomarkers has been associated with poorer prognosis, they have yet to be applied clinically. There are no standardized techniques or clinical values developed to assess the expression of these biomarkers in HNSCC tumor samples. HPV status is unique among this group as it is now routinely tested in many patients with oropharyngeal tumors and carries with it an improved prognosis. Methods such as protein and DNA microarray technology can be used to assess the molecular profile of a tumor and test for multiple biomarkers simultaneously [80]. Thus far, microarrays have not been applied outside the laboratory setting in HNSCC.

EGFR expression, and its inhibition with antibodies such as cetuximab, presents an example of how biomarkers can be successfully used as indicators of prognosis and as therapeutic targets to improve survival. VEGF is likely the next biomarker to be targeted in HNSCC therapy in daily clinical practice. The tumor suppressor genes $\mathrm{p} 53$ and $\mathrm{Rb}$, and regulatory proteins like SphK1 and EphB4/EphrinB2 offer both prognostic value and a means with which to detect malignant cells. However, the presence of these biomarkers is not routinely tested for in the clinical laboratory and therapies targeting these biomarkers have yet to be developed.

While there has been a great deal of advancement in biomarker detection and targeted therapies in HNSCC, there are still very important aspects of HNSCC we do not understand. In patients with HNSCC, there is an approximate $4 \%$ annual risk of developing a second primary tumor [81]. These second primary tumors are thought to be a result of "field cancerization" $[82,83]$. A future area of interest would be to identify a biomarker of field cancerization and develop a target that would prevent recurrence or a second primary tumor.
HNSCC is a tumor that carries with it a significant morbidity and a very poor prognosis, especially in advanced disease. Therefore, the development of biomarkers that can play a role in the earlier detection of tumor cells, offer prognostic information and can be used as targeted therapies is crucial.

\section{References}

[1] E. E. Vokes, R. R. Weichselbaum, S. M. Lippman et al., "Head and neck cancer," New England Journal of Medicine, vol. 328, no. 3, pp. 184-194, 1993.

[2] R. Sankaranarayanan, E. Masuyer, R. Swaminathan, J. Ferlay, and S. Whelan, "Head and neck cancer: a global perspective on epidemiology and prognosis," Anticancer Research, vol. 18, no. 6 B, pp. 4779-4786, 1998.

[3] K. Shibuya, C. D. Mathers, C. Boschi-Pinto, A. D. Lopez, and C. J. L. Murray, "Global and regional estimates of cancer mortality and incidence by site: II. Results for the global burden of disease 2000," BMC Cancer, vol. 2, no. 1, article 37, 2002.

[4] A. Jemal, R. Siegel, J. Xu, and E. Ward, "Cancer statistics, 2010," CA Cancer Journal for Clinicians, vol. 60, no. 5, pp. 277$300,2010$.

[5] A. Forastiere, W. Koch, A. Trotti, and D. Sidransky, "Head and neck cancer," New England Journal of Medicine, vol. 345, no. 26, pp. 1890-1900, 2001.

[6] J. Califano, W. H. Westra, G. Meininger, R. Corio, W. M. Koch, and D. Sidransky, "Genetic progression and clonal relationship of recurrent premalignant head and neck lesions," Clinical Cancer Research, vol. 6, no. 2, pp. 347-352, 2000.

[7] D. M. Shin, J. S. Lee, and L. G. Choi, "Prognostic significance of p53 expression in head and neck Squamous cell carcinoma," Proceedings of the American Society of Clinical Oncology, vol. 13, pp. 283-289, 1994.

[8] M. L. Poeta, J. Manola, M. A. Goldwasser et al., “TP53 mutations and survival in squamous-cell carcinoma of the head and neck," New England Journal of Medicine, vol. 357, no. 25, pp. 2552-2561, 2007.

[9] M. V. Gonzalez, M. P. Pello, C. Lopez-Larrea, C. Suarez, M. J. Menendez, and E. Coto, "Loss of heterozygosity and mutation analysis of the p16 (9p21) and p53 (17p13) genes in squamous cell carcinoma of the head and neck," Clinical Cancer Research, vol. 1, no. 9, pp. 1043-1049, 1995.

[10] J. A. Brennan, J. O. Boyle, W. M. Koch et al., "Association between cigarette smoking and mutation of the p53 gene in squamous-cell carcinoma of the head and neck," New England Journal of Medicine, vol. 332, no. 11, pp. 712-717, 1995.

[11] L. Mao, J. S. Lee, Y. H. Fan et al., "Frequent microsatellite alterations at chromosomes 9p21 and 3p14 in oral premalignant lesions and their value in cancer risk assessment," Nature Medicine, vol. 2, no. 6, pp. 682-685, 1996.

[12] D. J. Lee, F. Schönleben, V. E. Banuchi et al., "Multiple tumorsuppressor genes on chromosome $3 p$ contribute to head and neck squamous cell carcinoma tumorigenesis," Cancer Biology and Therapy, vol. 10, no. 7, pp. 689-693, 2010.

[13] http://www.clinicaltrials.gov/.

[14] M. P. Rosin, X. Cheng, C. Poh et al., "Use of allelic loss to predict malignant risk for low-grade oral epithelial dysplasia," Clinical Cancer Research, vol. 6, no. 2, pp. 357-362, 2000.

[15] J. J. Lee, W. K. Hong, W. N. Hittelman et al., "Predicting cancer development in oral leukoplakia: ten years of translational research," Clinical Cancer Research, vol. 6, no. 5, pp. 1702-1710, 2000. 
[16] O. Cuvillier, G. Pirianov, B. Kleuser et al., "Suppression of ceramide-mediated programmed cell death by sphingosine- 1phosphate," Nature, vol. 381, no. 6585, pp. 800-803, 1996.

[17] T. Kohama, A. Olivera, L. Edsall, M. M. Nagiec, R. Dickson, and S. Spiegel, "Molecular cloning and functional characterization of murine sphingosine kinase," Journal of Biological Chemistry, vol. 273, no. 37, pp. 23722-23728, 1998.

[18] S. M. Mandala, R. Thornton, Z. Tu et al., "Sphingoid base 1-phosphate phosphatase: a key regulator of sphingolipid metabolism and stress response," Proceedings of the National Academy of Sciences of the United States of America, vol. 95, no. 1, pp. 150-155, 1998.

[19] A. Olivera, T. Kohama, L. Edsall et al., "Sphingosine kinase expression increases intracellular sphingosine-1- phosphate and promotes cell growth and survival," Journal of Cell Biology, vol. 147, no. 3, pp. 545-557, 1999.

[20] S. Spiegel and S. Milstien, "Functions of the multifaceted family of sphingosine kinases and some close relatives," Journal of Biological Chemistry, vol. 282, no. 4, pp. 2125-2129, 2007.

[21] U. K. Sinha, V. J. Schorn, C. Hochstim, S. B. Chinn, S. Zhu, and R. Masood, "Increased radiation sensitivity of head and neck squamous cell carcinoma with sphingosine kinase 1 inhibition," Head and Neck, vol. 33, no. 2, pp. 178-188, 2011.

[22] S. Kalyankrishna and J. R. Grandis, "Epidermal growth factor receptor biology in head and neck cancer," Journal of Clinical Oncology, vol. 24, no. 17, pp. 2666-2672, 2006.

[23] Y. Yarden, "The EGFR family and its ligands in human cancer: signalling mechanisms and therapeutic opportunities," European Journal of Cancer, vol. 37, supplement 4, pp. S3-S8, 2001.

[24] C. Kari, T. O. Chan, M. Rocha de Quadros, and U. Rodeck, "Targeting the epidermal growth factor receptor in cancer: apoptosis takes center stage," Cancer Research, vol. 63, no. 1, pp. 1-5, 2003.

[25] X. H. Peng, P. Karna, Z. Cao, B. H. Jiang, M. Zhou, and L. Yang, "Cross-talk between epidermal growth factor receptor and hypoxia-inducible factor- $1 \alpha$ signal pathways increases resistance to apoptosis by up-regulating survivin gene expression," Journal of Biological Chemistry, vol. 281, no. 36, pp. 25903-25914, 2006.

[26] A. Psyrri, Z. Yu, P. M. Weinberger et al., "Quantitative determination of nuclear and cytoplasmic epidermal growth factor receptor expression in oropharyngeal squamous cell cancer by using automated quantitative analysis," Clinical Cancer Research, vol. 11, no. 16, pp. 5856-5862, 2005.

[27] K. K. Ang, B. A. Berkey, X. Tu et al., "Impact of epidermal growth factor receptor expression on survival and pattern of relapse in patients with advanced head and neck carcinoma," Cancer Research, vol. 62, no. 24, pp. 7350-7356, 2002.

[28] J. R. Grandis, M. F. Melhem, W. E. Gooding et al., "Levels of TGF- $\alpha$ and EGFR protein in head and neck squamous cell carcinoma and patient survival," Journal of the National Cancer Institute, vol. 90, no. 11, pp. 824-832, 1998.

[29] H. Sunada, B. E. Magun, J. Mendelsohn, and C. L. MacLeod, "Monoclonal antibody against epidermal growth factor receptor is internalized without stimulating receptor phosphorylation," Proceedings of the National Academy of Sciences of the United States of America, vol. 83, no. 11, pp. 3825-3829, 1986.

[30] M. L. Jaramillo, Z. Leon, S. Grothe, B. Paul-Roc, A. Abulrob, and M. O'Connor McCourt, "Effect of the anti-receptor ligand-blocking 225 monoclonal antibody on EGF receptor endocytosis and sorting," Experimental Cell Research, vol. 312, no. 15 , pp. $2778-2790,2006$.
[31] M. Prewett, P. Rockwell, C. Rose, and N. I. Goldstein, "Antitremor and cell cycle responses in $\mathrm{KB}$ cells treated with a chimeric anti-EGFR monoclonal antibody in combination with cisplatin," International Journal of Oncology, vol. 9, no. 2, pp. 217-224, 1996.

[32] J. Mendelsohn, "Epidermal growth factor receptor inhibition by a monoclonal antibody as anticancer therapy," Clinical Cancer Research, vol. 3, no. 12, pp. 2703-2707, 1997.

[33] R. C. Lilenbaum, B. Johnson, T. Lynch et al., "The evolving role of cetuximab in non-small cell lung cancer," Clinical Cancer Research, vol. 12, no. 14, pp. 4432s-4435s, 2006.

[34] J. A. Bonner, P. M. Harari, J. Giralt et al., "Radiotherapy plus cetuximab for squamous-cell carcinoma of the head and neck," New England Journal of Medicine, vol. 354, no. 6, pp. 567-578, 2006.

[35] S. Kim, J. R. Grandis, A. Rinaldo, R. P. Takes, and A. Ferlito, "Emerging perspectives in epidermal growth factor receptor targeting in head and neck cancer," Head and Neck, vol. 30, no. 5, pp. 667-674, 2008.

[36] J. B. Vermorken, R. Mesia, F. Rivera et al., "Platinum-based chemotherapy plus cetuximab in head and neck cancer," New England Journal of Medicine, vol. 359, no. 11, pp. 1116-1127, 2008.

[37] E. E. W. Cohen, F. Rosen, W. M. Stadler et al., "Phase II trial of ZD1839 in recurrent or metastatic squamous cell carcinoma of the head and neck," Journal of Clinical Oncology, vol. 21, no. 10, pp. 1980-1987, 2003.

[38] R. H. Wheeler, D. Jones, P. Sharma et al., "Clinical and molecular phase II study of gefitinib in patients (pts) with recurrent squamous cell cancer of the head and neck (H\&N Ca)," Journal of Clinical Oncology, vol. 23, article 5531, 2005.

[39] E. E. W. Cohen, M. A. Kane, M. A. List et al., "Phase II trial of gefitinib $250 \mathrm{mg}$ daily in patients with recurrent and/or metastatic squamous cell carcinoma of the head and neck," Clinical Cancer Research, vol. 11, no. 23, pp. 8418-8424, 2005.

[40] D. Soulieres, N. N. Senzer, E. E. Vokes, M. Hidalgo, S. S. Agarvala, and L. L. Siu, "Multicenter phase II study of erlotinib, an oral epidermal growth factor receptor tyrosine kinase inhibitor, in patients with recurrent or metastatic squamous cell cancer of the head and neck," Journal of Clinical Oncology, vol. 22, no. 1, pp. 77-85, 2004.

[41] L. L. Siu, D. Soulieres, E. X. Chen et al., "Phase I/II trial of erlotinib and cisplatin in patients with recurrent or metastatic squamous cell carcinoma of the head and neck: a Princess Margaret Hospital Phase II Consortium and National Cancer Institute of Canada Clinical Trials Group study," Journal of Clinical Oncology, vol. 25, no. 16, pp. 2178-2183, 2007.

[42] P. U. Hegde, A. C. Brenski, D. D. Caldarelli et al., "Tumor angiogenesis and p53 mutations: prognosis in head and neck cancer," Archives of Otolaryngology, vol. 124, no. 1, pp. 80-85, 1998.

[43] J. Folkman, "What is the evidence that tumors are angiogenesis dependent?" Journal of the National Cancer Institute, vol. 82, no. 1, pp. 4-6, 1990.

[44] J. Folkman, "Angiogenesis in cancer, vascular, rheumatoid and other disease," Nature Medicine, vol. 1, no. 1, pp. 27-31, 1995.

[45] J. Folkman, "Clinical applications of research on angiogenesis," New England Journal of Medicine, vol. 333, no. 26, pp. 1757-1763, 1995.

[46] J. Folkman and D. Hanahan, "Switch to the angiogenic phenotype during tumorigenesis," Princess Takamatsu Symposia, vol. 22, pp. 339-347, 1991.

[47] N. W. Gale and G. D. Yancopoulos, "Growth factors acting via endothelial cell-specific receptor tyrosine kinases: VEGFs, 
angiopoietins, and ephrins in vascular development," Genes and Development, vol. 13, no. 9, pp. 1055-1066, 1999.

[48] P. A. Kyzas, I. W. Cunha, and J. P. A. Ioannidis, "Prognostic significance of vascular endothelial growth factor immunohistochemical expression in head and neck squamous cell carcinoma: a meta-analysis," Clinical Cancer Research, vol. 11, no. 4, pp. 1434-1440, 2005.

[49] L. Strauss, D. Volland, M. Kunkel, and T. E. Reichert, "Dual role of VEGF family members in the pathogenesis of head and neck cancer (HNSCC): possible link between angiogenesis and immune tolerance," Medical Science Monitor, vol. 11, no. 8, pp. BR280-BR292, 2005.

[50] B. D. Smith, G. L. Smith, D. Carter, C. T. Sasaki, and B. G. Haffty, "Prognostic significance of Vascular Endothelial Growth Factor protein levels in oral and oropharyngeal squamous cell carcinoma," Journal of Clinical Oncology, vol. 18, no. 10, pp. 2046-2052, 2000.

[51] J. P. H. Machiels, S. Henry, S. Zanetta et al., "Phase II study of sunitinib in recurrent or metastatic squamous cell carcinoma of the head and neck: GORTEC 2006-01," Journal of Clinical Oncology, vol. 28, no. 1, pp. 21-28, 2010.

[52] C. Elser, L. L. Siu, E. Winquist et al., "Phase II trial of sorafenib in patients with recurrent or metastatic squamous cell carcinoma of the head and neck or nasopharyngeal carcinoma," Journal of Clinical Oncology, vol. 25, no. 24, pp. 3766-3773, 2007.

[53] M. G. Fury, A. Zahalsky, R. Wong et al., "A Phase II study of SU5416 in patients with advanced or recurrent head and neck cancers," Investigational New Drugs, vol. 25, no. 2, pp. 165172, 2007.

[54] T. Y. Seiwert, D. J. Haraf, E. E. W. Cohen et al., "Phase I study of bevacizumab added to fluorouracil- and hydroxyurea-based concomitant chemoradiotherapy for poor-prognosis head and neck cancer," Journal of Clinical Oncology, vol. 26, no. 10, pp. 1732-1741, 2008.

[55] E. E. Cohen, D. W. Davis, T. G. Karrison et al., "Erlotinib and bevacizumab in patients with recurrent or metastatic squamous-cell carcinoma of the head and neck: a phase I/II study," The Lancet Oncology, vol. 10, no. 3, pp. 247-257, 2009.

[56] D. Sano, D. R. Fooshee, M. Zhao et al., "Targeted molecular therapy of head and neck squamous cell carcinoma with the tyrosine kinase inhibitor vandetanib in a mouse model," Head and Neck, vol. 33, no. 3, pp. 349-358, 2011.

[57] N. Holder and R. Klein, "Eph receptors and ephrins: effectors of morphogenesis," Development, vol. 126, no. 10, pp. 20332044, 1999.

[58] E. B. Pasquale, “The Eph family of receptors," Current Opinion in Cell Biology, vol. 9, no. 5, pp. 608-615, 1997.

[59] S. S. Gerety, H. U. Wang, Z. F. Chen, and D. J. Anderson, "Symmetrical mutant phenotypes of the receptor EphB4 and its specific transmembrane ligand ephrin-B2 in cardiovascular development," Molecular Cell, vol. 4, no. 3, pp. 403-414, 1999.

[60] E. J. Yavrouian, U. K. Sinha, D. H. Rice, M. T. Salam, P. S. Gill, and R. Masood, "The significance of EphB4 and EphrinB2 expression and survival in head and neck squamous cell carcinoma," Archives of Otolaryngology, vol. 134, no. 9, pp. 985991, 2008.

[61] R. Masood, S. Ram Kumar, U. K. Sinha et al., "EphB4 provides survival advantage to squamous cell carcinoma of the head and neck," International Journal of Cancer, vol. 119, no. 6, pp. 1236-1248, 2006.

[62] U. K. Sinha, K. Mazhar, S. B. Chinn et al., "The association between elevated EphB4 expression, smoking status, and advanced-stage disease in patients with head and neck squamous cell carcinoma," Archives of Otolaryngology, vol. 132, no. 10, pp. 1053-1059, 2006.

[63] U. K. Sinha, A. Kundra, P. Scalia et al., "Expression of EphB4 in head and neck squamous cell carcinoma," Ear, Nose and Throat Journal, vol. 82, no. 11, pp. 866-887, 2003.

[64] M. J. Lace, J. R. Anson, J. P. Klussmann et al., "Human papillomavirus type 16 (HPV-16) genomes integrated in head and neck cancers and in HPV-16-immortalized human keratinocyte clones express chimeric virus-cell mRNAs similar to those found in cervical cancers," Journal of Virology, vol. 85, no. 4, pp. 1645-1654, 2011.

[65] M. L. Gillison, W. M. Koch, R. B. Capone et al., "Evidence for a causal association between human papillomavirus and a subset of head and neck cancers," Journal of the National Cancer Institute, vol. 92, no. 9, pp. 709-720, 2000.

[66] A. R. Kreimer, G. M. Clifford, P. Boyle, and S. Franceschi, "Human papillomavirus types in head and neck squamous cell carcinomas worldwide: a systemic review," Cancer Epidemiology Biomarkers and Prevention, vol. 14, no. 2, pp. 467-475, 2005.

[67] M. E. McLaughlin-Drubin and K. Münger, "Oncogenic activities of human papillomaviruses," Virus Research, vol. 143, no. 2, pp. 195-208, 2009.

[68] T. D. Kessis, R. J. Slebos, W. G. Nelson et al., "Human papillomavirus 16 E6 expression disrupts the p53-mediated cellular response to DNA damage," Proceedings of the National Academy of Sciences of the United States of America, vol. 90, no. 9, pp. 3988-3992, 1993.

[69] K. Huh, X. Zhou, H. Hayakawa et al., "Human papillomavirus type 16 E7 oncoprotein associates with the cullin 2 ubiquitin ligase complex, which contributes to degradation of the retinoblastoma tumor suppressor," Journal of Virology, vol. 81, no. 18, pp. 9737-9747, 2007.

[70] A. M. De Roda Husman, J. M. M. Walboomers, A. J. C. Van den Brule, C. J. L. M. Meijer, and P. J. F. Snijders, "The use of general primers GP5 and GP6 elongated at their 3' ends with adjacent highly conserved sequences improves human papillomavirus detection by PCR," Journal of General Virology, vol. 76, no. 4, pp. 1057-1062, 1995.

[71] M. L. Gillison, J. Harris, and W. Westra, "Survival outcomes by tumor human papillomavirus (HPV) status in stage IIIIV oropharyngeal cancer (OPC) in RTOG 0129," Proceedings of the American Society of Clinical Oncology, vol. 27, no. 15S, article 6003, 2009.

[72] C. Fakhry, W. H. Westra, S. Li et al., "Improved survival of patients with human papillomavirus-positive head and neck squamous cell carcinoma in a prospective clinical trial," Journal of the National Cancer Institute, vol. 100, no. 4, pp. 261269, 2008.

[73] D. Lindquist, M. Romanitan, L. Hammarstedt et al., "Human papillomavirus is a favourable prognostic factor in tonsillar cancer and its oncogenic role is supported by the expression of E6 and E7," Molecular Oncology, vol. 1, no. 3, pp. 350-355, 2007.

[74] L. Licitra, F. Perrone, P. Bossi et al., "High-risk human papillomavirus affects prognosis in patients with surgically treated oropharyngeal squamous cell carcinoma," Journal of Clinical Oncology, vol. 24, no. 36, pp. 5630-5636, 2006.

[75] http://www.cdc.gov/.

[76] A. Wu, Q. Zeng, T. H. Kang et al., "Innovative DNA vaccine for human papillomavirus (HPV)-associated head and neck cancer," Gene Therapy, vol. 18, pp. 304-312, 2011.

[77] S. Sethi, M. S. Benninger, M. Lu, S. Havard, and M. J. Worsham, "Noninvasive molecular detection of head and neck 
squamous cell carcinoma: an exploratory analysis," Diagnostic Molecular Pathology, vol. 18, no. 2, pp. 81-87, 2009.

[78] J. Califano, S. A. Ahrendt, G. Meininger, W. H. Westra, W. M. Koch, and D. Sidransky, "Detection of telomerase activity in oral rinses from head and neck squamous cell carcinoma patients," Cancer Research, vol. 56, no. 24, pp. 5720-5722, 1996.

[79] A. Matta, R. Ralhan, L. V. Desouza, and K. W. M. Siu, "Mass spectrometry-based clinical proteomics: head-and-neck cancer biomarkers and drug-targets discovery," Mass Spectrometry Reviews, vol. 29, no. 6, pp. 945-961, 2010.

[80] R. Radhakrishnan, M. Solomon, K. Satyamoorthy, L. E. Martin, and M. W. Lingen, "Tissue microarray-a high-throughput molecular analysis in head and neck cancer," Journal of Oral Pathology and Medicine, vol. 37, no. 3, pp. 166-176, 2008.

[81] X. León, M. Quer, S. Diez, C. Orús, A. López-Pousa, and J. Burgués, "Second neoplasm in patients with head and neck cancer," Head and Neck, vol. 21, no. 3, pp. 204-210, 1999.

[82] D. P. Slaughter, H. W. Southwick, and W. Smejkal, "Field cancerization in oral stratified squamous epithelium. Clinical implications of multicentric origin," Cancer, vol. 6, pp. 963-968, 1953.

[83] E. Rennemo, U. Zätterström, and M. Boysen, "Impact of second primary tumors on survival in head and neck cancer: an analysis of 2,063 cases," Laryngoscope, vol. 118, no. 8, pp. 13501356, 2008. 


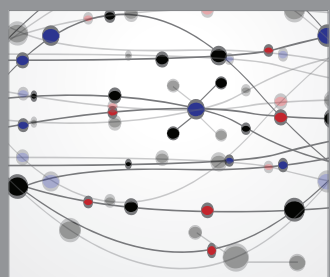

The Scientific World Journal
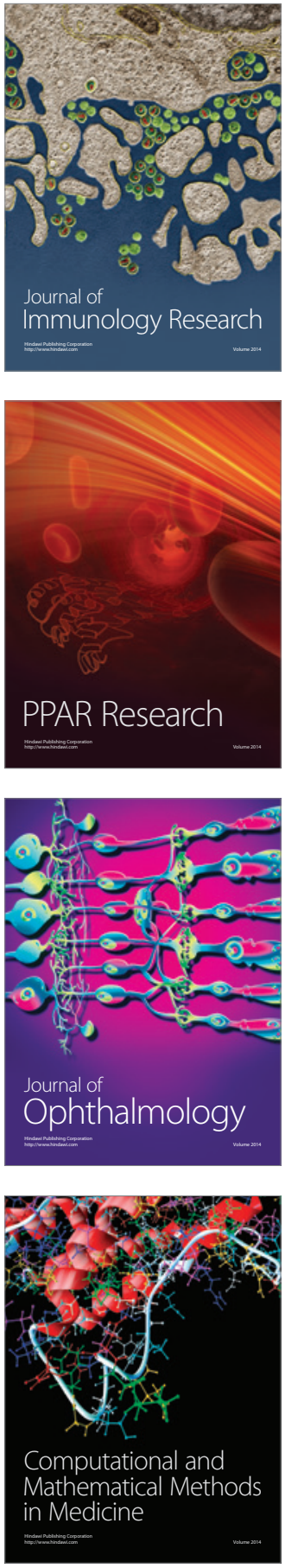

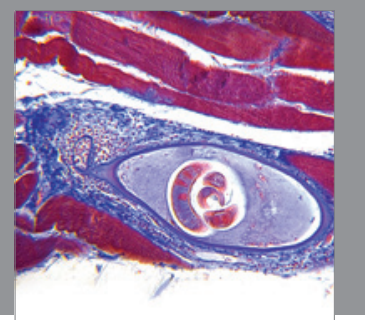

Gastroenterology

Research and Practice
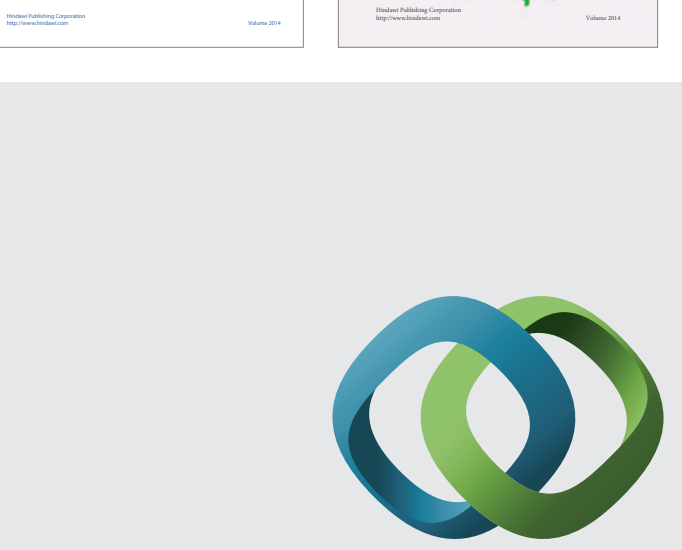

\section{Hindawi}

Submit your manuscripts at

http://www.hindawi.com
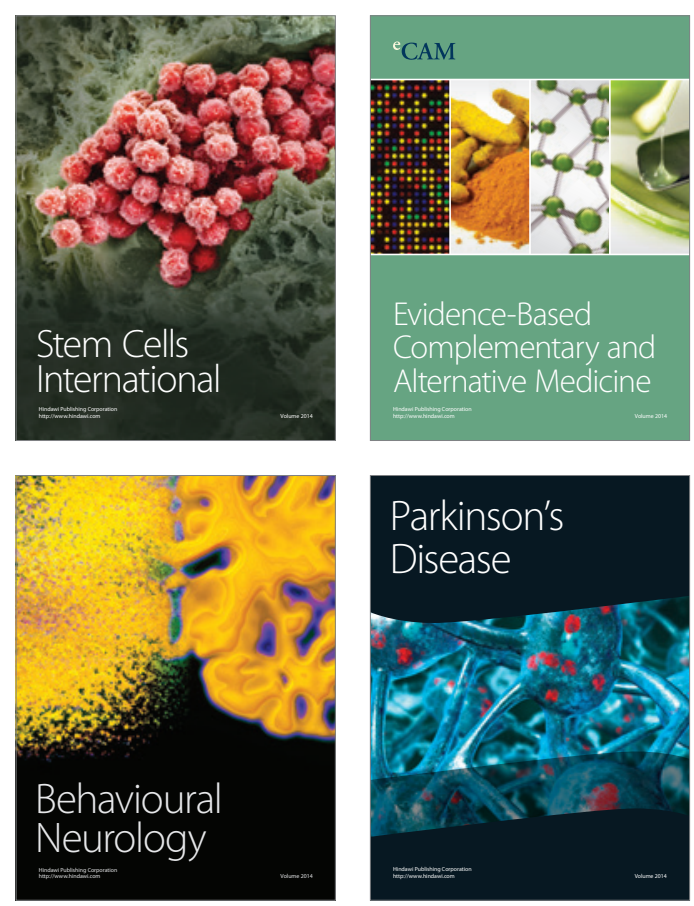

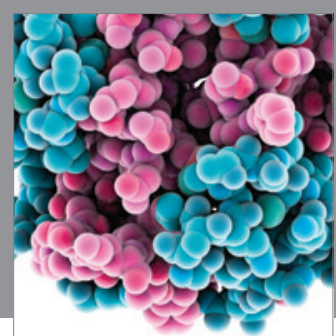

Journal of
Diabetes Research

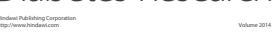

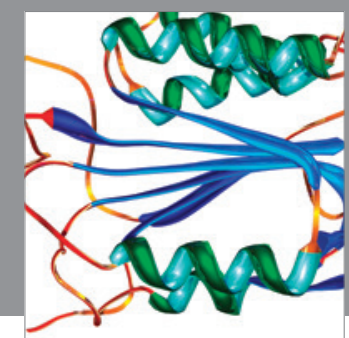

Disease Markers
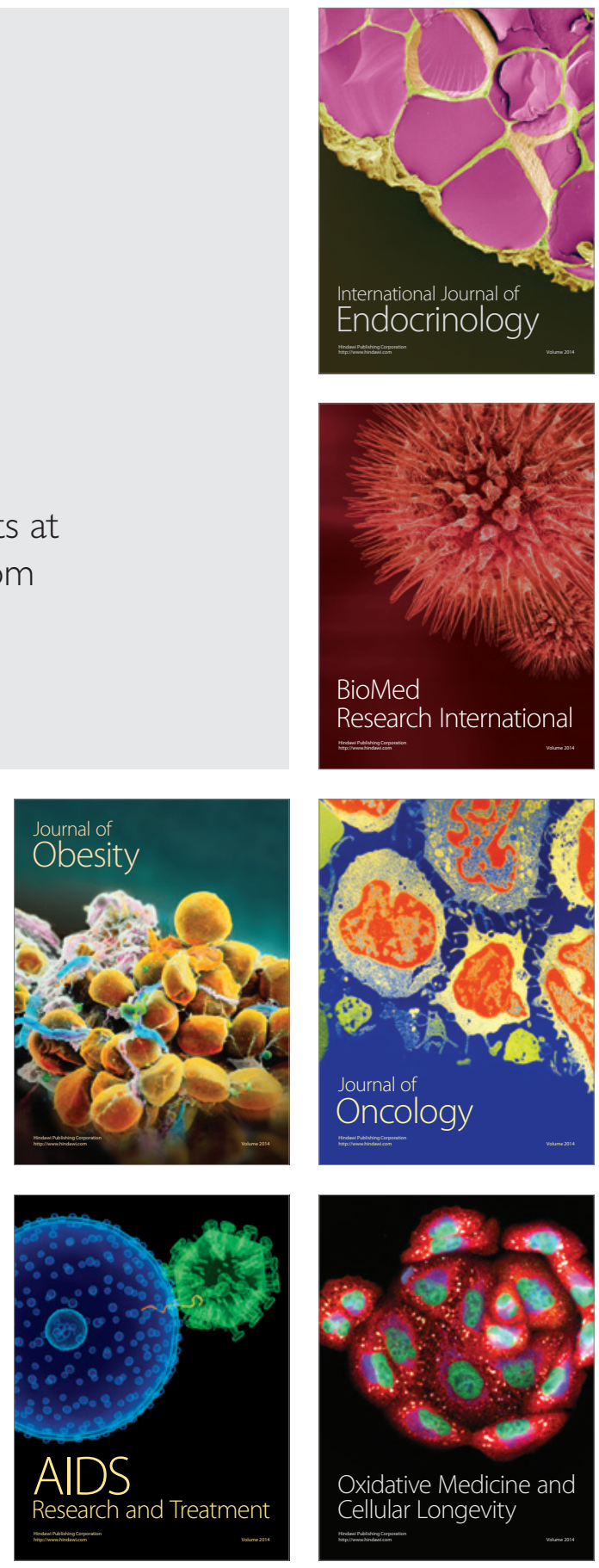